\title{
Paclitaxel and cisplatin chemotherapy for ovarian cancer during pregnancy: case report and review of the literature
}

\author{
Krystyna Serkies $\cdot$ Ewa Węgrzynowicz $\cdot$ Jacek Jassem
}

Received: 18 June 2010 / Accepted: 1 February 2011 / Published online: 3 March 2011

(C) The Author(s) 2011. This article is published with open access at Springerlink.com

\begin{abstract}
The safety of chemotherapy during pregnancy is debatable. We present a case of advanced ovarian cancer, diagnosed at week 28 of gestational age, treated with 2 cycles of paclitaxel/cisplatin (TC) chemotherapy during pregnancy, with no serious toxicity. At week 34, the patient underwent a caesarean section and delivered a healthy girl. Four additional cycles of TC were administered. Three months after completing chemotherapy, the patient developed abdominal progression and subsequently a huge metastatic cystic mass in the brain. Despite subsequent therapies, the patient died of progressive disease 35 months after the diagnosis. The infant had normal growth and development by 73 months of her age. This is another reported case of ovarian cancer diagnosed during the second trimester of the pregnancy treated with TC chemotherapy without apparent teratogenic effect.
\end{abstract}

Keywords Pregnancy - Ovarian carcinoma ·

Chemotherapy

\section{Introduction}

Diagnosis of cancer during pregnancy is uncommon, with breast and cervical cancer, lymphoma, and melanoma being the most frequent malignancies. Nevertheless, the incidence of cancer in pregnancy is increasing, probably due to routine use of sonography during prenatal care and the ten-

K. Serkies $(\varangle) \cdot$ E. Węgrzynowicz $\cdot$ J. Jassem Department of Oncology and Radiotherapy, Medical University of Gdańsk, 7 Dębinki St., 80-211 Gdańsk, Poland e-mail: onkol@gumed.edu.pl dency to postpone childbirth to an older age. The standard therapy for advanced epithelial ovarian cancer (EOC) consists of cytoreductive surgery followed by chemotherapy, preferably including paclitaxel combined with cisplatin or carboplatin. We present here a case of a pregnant woman with advanced EOC, treated with paclitaxel/cisplatin (TC) chemotherapy, with preservation of the pregnancy.

\section{A case}

A 24-year-old woman, gravida 1, presented with an asymptomatic polycystic adnexal mass diagnosed at week 28 of gestation at the routine ultrasound examination. The patient had no history of ovarian, colon or breast cancer in the family. She underwent a laparotomy with bilateral salpingooophorectomy, omentectomy and appendectomy. Intraoperative examination showed disseminated small multiple peritoneal nodules and a few superficial liver metastases up to $2 \mathrm{~cm}$ in size. The postoperative course was uneventful. The abdomen ultrasound performed postoperatively confirmed the presence of metastatic liver lesions sized 32$80 \mathrm{~mm}$, located in both lobes. The final diagnosis was stage IV, grade 2 mucinous ovarian adenocarcinoma. After extensive discussion with the patient and her family, informed consent was obtained for postoperative chemotherapy, with attempted preservation of the pregnancy. Nineteen days after the surgery, chemotherapy consisting of paclitaxel $175 \mathrm{mg} / \mathrm{m}^{2}$ over $3 \mathrm{~h}$ and cisplatin $75 \mathrm{mg} / \mathrm{m}^{2}$, with standard premedication of steroids and anti-emetics (20 mg dexamethason twice a day, $8 \mathrm{mg}$ ondansetrone) was initiated. The baseline serum liver and renal function tests were normal; the level of $\mathrm{Ca} 125$ before starting chemotherapy was $71 \mathrm{IU} / \mathrm{ml}$. After 3 weeks, the patient received a second chemotherapy cycle. Treatment was well tolerated. 
At week 34, after administration of corticosteroids for foetal lung maturation, a caesarean section with staging laparotomy was performed. The exploration of the abdomen cavity showed multiple small persisted post-chemotherapy lesions located in the perimetrium, peritoneum of vesicouterine fold, Douglas sinus and the diaphragmatic dome, as well as metastatic lesions on the liver surface of $0.1-1 \mathrm{~cm}$ in diameter. The woman delivered a healthy girl $(1,900 \mathrm{~g}$, Apgar score 8 at $5 \mathrm{~min}$, and normal laboratory tests). There were no peri- or postoperative complications. Four additional TC cycles were administered. Ca 125 level after completing the sixth cycle of chemotherapy was $39 \mathrm{IU} / \mathrm{ml}$. Progressive disease in the liver (tumour lesions of $55 \times 46 \mathrm{~mm}$ in size) and enlarged para-aortic lymph nodes were found on CT scans performed 3 months later. Subsequent 7 cycles of topotecan resulted in disease stabilization and $\mathrm{Ca} 125$ level was $30 \mathrm{IU} / \mathrm{ml}$. Topotecan administration was stopped after a huge cystic mass $(55 \times 45 \times 50 \mathrm{~mm})$ in the brain had been diagnosed, following headache complaints. Negative cytology was obtained after diagnostic biopsy. The patient received palliative whole brain irradiation of 20 Gy in 5 fractions followed by chemotherapy: subsequently single-agent carboplatin, cyclophosphamide/ cisplatin and finally 2 cycles of liposomal doxorubicin. Despite therapy, 35 months after cancer diagnosis, the patient died of progressive abdominal lesions without central nervous system symptoms. The infant had normal growth and development at 73 months of her age.

\section{Discussion}

The development of malignant disease during pregnancy leads to an enormous emotional burden to the patient, her partner and the medical staff, accompanied by medical dilemmas. Surgery for malignant tumours seems to be safe, whereas the teratogenicity of cytotoxic agents depends on several factors, including their type, timing of exposure, dose and characteristics affecting placental transfer [1]. The use of chemotherapy during the first trimester increases the risk of spontaneous abortion, foetal death and major malformation and is generally contraindicated in this period. Accumulating data suggests that at the time organogenesis is completed, the risk of intrauterine growth restriction and low birth-weight is low. In consequence, chemotherapy is a viable option in selected pregnant women. However, only a few studies have evaluated the long-term consequences for children exposed to chemotherapy while in utero [1-5]. Owing to the limited clinical experience and the unique taxane antineoplastic mode of action, generally the use of taxanes in pregnancy is not recommended [1]. Until now, only a few cases of chemotherapy with taxanes, including paclitaxel alone or in combination with either cisplatin or carbo- platin, applied during pregnancy, have been reported [1, 3 , 4, 6-14]. In all cases but one, organogenesis had been completed by the time of treatment onset. Current experience includes five cases of EOC treated with 6, 5 (two cases), 4 and 3 cycles of paclitaxel-based chemotherapy, respectively, and one patient with FIGO III dysgerminoma administered 4 cycles of paclitaxel/carboplatin (Table 1) [6, 9-12, 14]. We presented another patient with advanced EOC, who received 2 cycles of TC chemotherapy during the second trimester of pregnancy. Therapy did not apparently affect the development of the infant during the first 6 years of observation. No teratogenic effects of 3 cycles of TC combination (with paclitaxel administered at $135 \mathrm{mg} / \mathrm{m}^{2} / 24 \mathrm{~h}$ ) for EOC during pregnancy were reported by Sood et al. [14] in 2001. Similarly to our case, the patient's outcome was fatal, whereas the growth and the development of the infant was normal at 29 months of age. No teratogenic effect was also shown within 20 months of the infant's life in the dysgerminoma patient administered paclitaxel/carboplatin chemotherapy [9]. In another case, the episode of respiratory insufficiency occurring immediately after the delivery in a newborn exposed to 3 cycles of TC chemotherapy due to mother's lung cancer was considered to be related to neonatal prematurity rather than chemotherapy toxicity [7].

Overall, there have been so far about 52 reports of pregnant women managed with platinum-based chemotherapy, including 19 women treated for EOC with 2-6 cycles of single-agent or cisplatin/carboplatin-containing multidrug chemotherapy, starting at the second or third trimester of gestation [3-6, 10-17].

Our patient was administered cisplatin as it is preferred over carboplatin owing to the higher risk of thrombocytopenia [1]. Additionally, in 2004 at the start of chemotherapy, the clinical experience on the use of carboplatin treatment in pregnant women was very limited.

The review of all studies (published in English from 1977 to March 2008) concerning platinum therapy showed 43 cases of pregnant women (cisplatin in 36 , carboplatin in 6 , and both agents in one patient) [5]. In this group two foetal malformations were recorded, in both cases after therapy with cisplatin. However, causality related to in utero cisplatin exposure could not be proven. Acute respiratory distress (in three premature newborns), cytopenia, elevation of serum creatinine and hearing impairment were also observed. No foetal malformations and normal neonatal examinations were reported after therapy with carboplatin. It is believed that the administration of both platinum salts is feasible during the second and third trimester of pregnancy; however owing to the transplacental transfer of these drugs in late pregnancy, collecting further data on teratogenic effects remains highly relevant.

Until now, the longest reported follow-up of the children exposed to intra-uterine chemotherapy with cisplatin or 


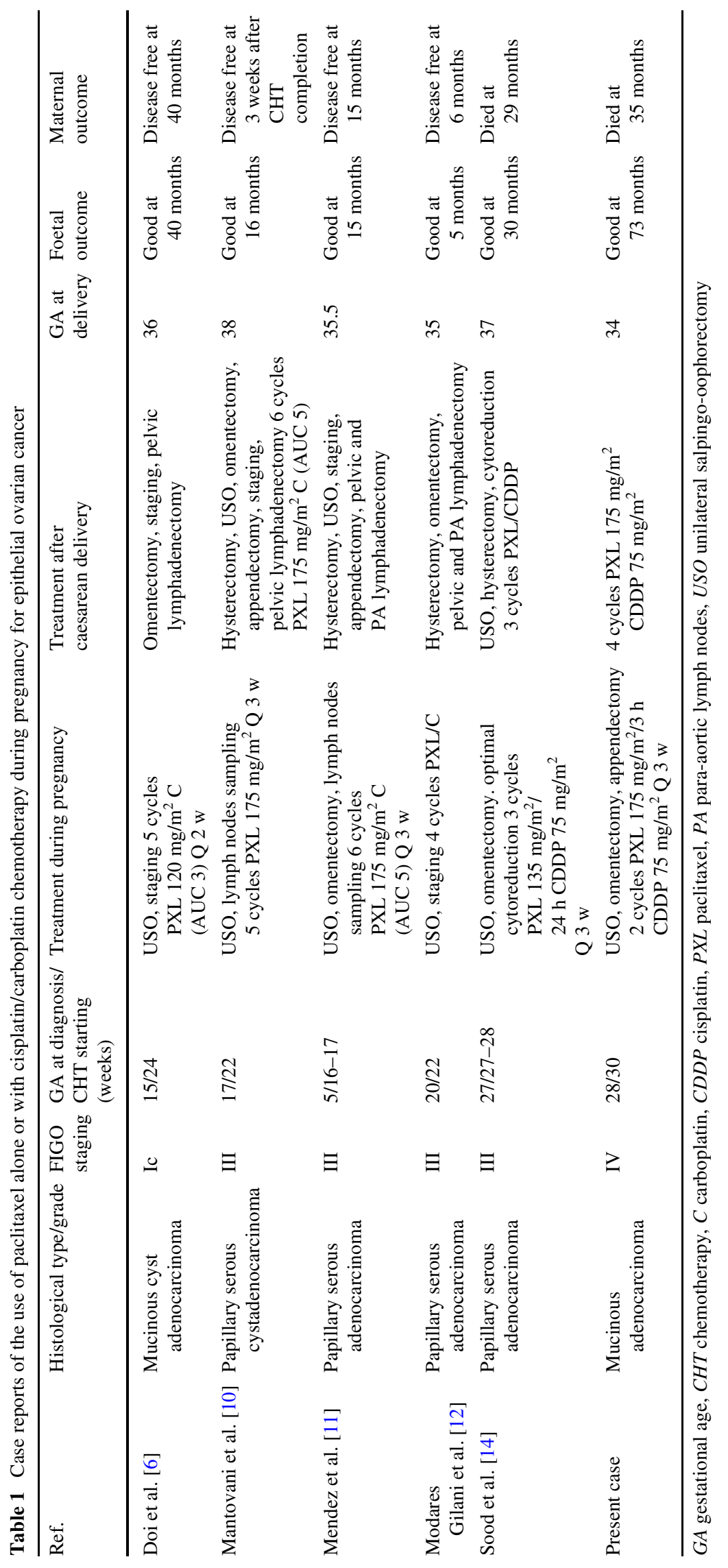


paclitaxel, with no evidence of sequelae, has been 42 months and 40 months, respectively [6, 15]. We have reported another case of a child exposed to the TC combination with no evidence of sequelae after 73 months of follow-up.

The staging and treatment of cancer at pregnancy should preferentially follow the standard approach [2]. Due to increased levels of Ca 125 in pregnancy and puerperium, the diagnostic value of this assay is limited [18]. The typical management of ovarian carcinoma includes total hysterectomy. Pregnant women diagnosed with advanced EOC should undergo organ-saving surgery, with the removal of the reproductive organs only after the delivery [19]. In our case, the surgery was restricted to a mere staging and this might have worsened treatment efficacy.

The prognosis of maternal ovarian cancers is similar to those in non-pregnant women in the reproductive age [20]. Suboptimal therapy and initial advanced stage of disease as a result of delayed diagnosis in some pregnant women are associated with worse outcome. The presented case was diagnosed in an advanced stage, with no real chance for cure.

In conclusion, standard chemotherapy including paclitaxel for advanced EOC diagnosed during pregnancy seems to be safe; however, potential risks and benefits of this therapy still have to be cautiously weighted.

\section{Conflict of interest None.}

Open Access This article is distributed under the terms of the Creative Commons Attribution Noncommercial License which permits any noncommercial use, distribution, and reproduction in any medium, provided the original author(s) and source are credited.

\section{References}

1. Cardonick E, Iacobucci A (2004) Use of chemotherapy during human pregnancy. Lancet Oncol 5:283-291

2. Amant F, Van Calsteren K, Halska MJ et al (2009) Gynecologic cancers in pregnancy: quidelines of an international consensus meeting. Int J Gynecol Cancer 19:1-12

3. Amant F, Brepoels L, Halaska MJ, Gziri MM, Calsteren KV (2010) Gynaecologic cancer complicating pregnancy: an overview. Best Pract Res Clin Obstet Gynaecol 24:61-79

4. Azim HA Jr, Peccatori FA, Pavlidis N (2010) Treatment of the pregnant mother with cancer: a systematic review on the use of cytotoxic, endocrine, targeted agents and immunotherapy during pregnancy. Part I: Solid tumors. Cancer Treat Rev 36:101-109
5. Mir O, Berveiller P, Ropert S, Goffinet F, Goldwasser F (2008) Use of platinum derivatives during pregnancy. Cancer 113:30693074

6. Doi D, Boh Y, Konishi H, Asakura H, Takeshita T (2009) Combined chemotherapy with paclitaxel and carboplatin for mucinous cystadenocarcinoma of the ovary during pregnancy. Arch Gynecol Obstet 280:633-636

7. Garcia-Gonzalez J, Cueva J, Lamas MJ, Curiel T, Grana B, LopezLopez R (2008) Paclitaxel and cisplatin in the treatment of metastatic non-small-cell lung cancer during pregnancy. Clin Transl Oncol 10:375-376

8. Gonzalez-Angulo AM, Walters RS, Carpenter RJ Jr et al (2004) Paclitaxel chemotherapy in a pregnant patient with bilateral breast cancer. Clin Breast Cancer 5:317-319

9. Hubalek M, Smekal-Schindelwig C, Zeimet AG et al (2007) Chemotherapeutic treament of a pregnant patient with ovarian dysgerminoma. Arch Gynecol Obstet 276:179-183

10. Mantovani G, Mais V, Parodo G, Carrucciu GM (2007) Use of chemotherapy for ovarian cancer during human pregnancy: case report and literature review. Eur J Obstet Gynecol Reprod Biol 131:235-245

11. Mendez LE, Mueller A, Salom E, Gonzalez-Quintero VH (2003) Paclitaxel and carboplatin chemotherapy administered during pregnancy for advanced epithelial ovarian cancer. Obstet Gynecol 102:1200-1202

12. Modares Gilani M, Karimi Zarchi M, Behtash N, Ghaemmaghami F, Mousari AS, Behnamfar E (2007) Preservation of pregnancy in a patient with advanced ovarian cancer at 20 weeks of gestation: case report and literature review. Int J Gynecol Cancer 17:1140 1143

13. Rouzi AA, Sahly NN, Sahly NF, Alahwal MS (2009) Cisplatinum and docetaxel for ovarian cancer in pregnancy. Arch Gynecol Obstet 280:823-825

14. Sood AK, Shahin MS, Sorosky JL (2001) Paclitaksel and platinum chemotherapy for ovarian carcinoma during pregnancy. Gynecol Oncol 83:599-600

15. Ferrandina G, Distefano M, Testa A, De Vincenzo R, Scambia G (2005) Management of an advanced ovarian cancer at 15 weeks of gestation: case report and literature review. Gynecol Oncol 97:693-696

16. Picone O, Lhomme C, Tournaire M, Pautier P, Camatte S, VacherLavanue MC (2004) Preservation of pregnancy in a patient with a stage IIIB ovarian epithelial carcinoma diagnosed at 22 weeks of gestation and treated with initial chemotherapy: case report and literature review. Gynecol Oncol 94:600-604

17. Tabata T, Nishiura K, Tanida K, Kondo E, Okugawa T, Sagawa N (2008) Carboplatin chemotherapy in a pregnant patient with undifferentiated ovarian carcinoma: case report and review of the literature. Int J Gynecol Cancer 18:181-184

18. Spitzer M, Kaushal N, Benjamin F (1998) Maternal Ca-125 levels in pregnancy and the puerperium. J Reprod Med 43:387-392

19. Marret H, Lhomme C, Lecuru F et al (2010) Guidelines for the management of ovarian cancer during pregnancy. Eur J Obstet Gynecol Reprod Biol 149:18-21

20. Zhao XY, Huang HF, Lian LJ, Lang JH (2006) Ovarian cancer in pregnancy: a clinicopathologic analysis of 22 cases and review of the literature. Int J Gynecol Cancer 16:8-15 